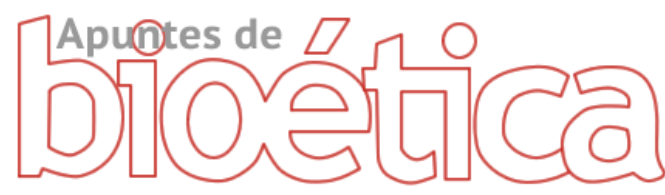

https://doi.org/10.35383/apuntes.v3i2.475

\title{
Relacionalidad e identidad: comprender el sentido existencial como vocación al amor
}

\author{
Risco Lázaro A. ${ }^{1}$
}

\section{INFORMACIÓN DEL ARTÍCULO RESUMEN}

\section{Historia del artículo:}

Recibido el 27 de setiembre de 2020

Aceptado el 29 de diciembre de 2020

\section{Palabras claves:}

Identidad

Interpersonalidad

Relacionalidad

Vocación al amor

Sentido existencial

En este trabajo se indaga en el aporte fundamental de las relaciones interpersonales en la configuración de la identidad personal, a partir de la consideración de la persona como ser esencialmente abierto y relacional, y del cuerpo como realidad simbólica de la vocación humana al amor. Comprendiendo que la identidad personal consiste en la autorrepresentación de sí mismo como ser singular y en homogeneidad con los semejantes, e identificándose a través de los continuos cambios y decisiones como un ser llamado al amor, su consecución requerirá un proceso gradual que abarca toda su existencia. Las etapas identitarias por las que avanza -filiación, esponsalidad, paternidad- requieren la adquisición de ciertas capacidades madurativas que procuren la experiencia de la acogida y la donación, elementos esenciales del amor. En el continuo de relaciones interpersonales en las que la persona se verá inmersa en el trayecto de su vida desde aquella primera que le dio origen, podrá elaborar la propia conciencia de sí y avanzar en su desarrollo mediante las habilidades psico-sociales que irá adquiriendo para afrontar las necesidades planteadas en cada etapa madurativa hasta lograr la consolidación de su propia identidad personal en la experiencia auténticamente humana del amor como sentido existencial.

Relationality and identity: understanding the existential meaning as a vocation to love

\section{ABSTRACT}

\section{Keywords:}

Identity

Relationality

Interpersonality

Vocation to the love

Existential meaning
This work investigates the fundamental contribution of interpersonal relationships in the configuration of personal identity, based on the consideration of the person as an essentially open and relational being, and of the body as a symbolic reality of the human vocation to love.

${ }^{1}$ Doctora en Psicología por la Universidad Pontificia de Salamanca: Salamanca, Castilla y León, Máster en Bioética por el Istituto Joanes Paulus II per studi su Matrimonio e familia, Master Universitario de segundo nivel en Bioetica e Formazione por la Università Cattolica del Sacro Cuore Sede di Roma. Docente de la Universidad de Valencia, Valencia España. Correo electrónico: ana.risco@ucv.es. ORCID: https://orcid.org/0000-0003-4925-1954 


\begin{abstract}
Understanding that personal identity consists of the self-representation of oneself as a singular being and in homogeneity with others and identifying through continuous changes and decisions as a being called to love, its achievement will require a gradual process that encompasses its entire existence. The identity stages through which it progresses - affiliation, nuptiality, paternity - require the acquisition of certain developmental capacities that provide the experience of welcoming and giving, essential elements of love. In the continuum of interpersonal relationships in which the person will be immersed in their own life journey from the first relationship with the one that gave birth to them, they will be able to develop their own self-awareness and advance in their development through the psychosocial skills that they will acquire to face the needs raised in each maturational stage until achieving the consolidation of their own personal identity in the authentically human experience of love as an existential meaning.
\end{abstract}

\section{Introducción}

La realidad humana se muestra como un profundo misterio cuando se trata de indagar en ella en busca de una comprensión integral en los distintos ámbitos de su existencia. La palabra misterio alude a algo que sobrepasa la mera evidencia posible a la propia lógica racional, lo cual suscita una actitud de profundo asombro, de la que emana el auténtico deseo de conocimiento.

Impulsados por tal actitud de asombro natural ante el misterio del ser humano, pretendemos adentrarnos en el estudio del desarrollo de la identidad personal. A este propósito pretendemos orientarnos desde una perspectiva concreta y del todo significativa: la de la interpersonalidad, comprendida como el auténtico encuentro entre personas libres en el amor (Pérez-Soba, 2005). Esto nos sitúa ante el hecho ineludible de la condición relacional humana y el papel prioritario de las relaciones interpersonales en su propio desarrollo y realización de su sentido existencial. Nos apoyamos para ello en la argumentación antropológica realizada por Martin Buber, conocido como "el filósofo del diálogo" y en las aportaciones científicas del psiquiatra Aquilino Polaino-Lorente sobre el estudio de la identidad personal y el proceso de su configuración, quien llama la atención sobre el papel prioritario del ámbito familiar y las primeras relaciones sociales que la persona establece. A partir de ello, indagaremos en la implicación del factor interpersonal en la progresiva conciencia de sí mismo y la realización de su llamada personal al amor a través de las distintas etapas evolutivas y las crisis correspondientes establecidas en la tradicional teoría del desarrollo psico-social formulada por Erik Erikson. De este modo, podremos comprender el alcance de las relaciones interpersonales en el desarrollo de la propia identidad personal y, en definitiva, en el sentido existencial.

Con todo, el objeto de este trabajo consiste en aportar una justificación de la 
importancia de la vida en relación como medio de desarrollo y realización personal, concretamente en la configuración de la propia identidad como núcleo fundamental de la persona. Esta reflexión nos parece del todo conveniente en el momento actual y es nuestro deseo que pudiera de algún modo arrojar claridad para la comprensión de la existencia humana, especialmente en la sociedad postmoderna marcada notablemente por el narcisismo, y en la cual la autocomplacencia y la autorreferencialidad son tomadas como criterio del actuar moral orientando a una idea vacua de la felicidad enmarcada en la esfera privada. Nos proponemos, en fin, contribuir a la recta valorización de la relacionalidad humana como elemento clave para la propia identidad personal, especialmente en este tiempo abocado al individualismo, a efectos de que redunde en la auténtica vida lograda de las personas.

\section{Dos premisas y un punto de partida}

\subsection{Dos premisas: ser abierto y relacional}

Al inicio de nuestro estudio, se nos plantea la necesidad de señalar dos premisas, extraídas de una visión intuitiva del ser humano, que sentarán las claves de interpretación del desarrollo posterior:

En primer lugar, la persona es un ser abierto, como afirman Altarejos y Naval (2004, p. 17):

el ser humano nace con una forma o modo de ser, como el resto de los vivientes, pero no nace formado.
Cualquier animal nace con unas tendencias definidas y completas, conformadas, que permanecerán a lo largo de su existencia [...] no es así en el ser humano; su nacimiento solo es la aparición de múltiples posibilidades que irán actualizándose o no, a lo largo de su existencia.

Se da en el ser humano una continua posibilidad creativa -la denominada plasticidad humana- por la que la persona es vulnerable a la realidad circundante de modo que, en cada situación vital, por la intervención de los distintos factores influyentes tanto ajenos como propios, puede decirse que la persona se está haciendo a sí misma.

Unido a lo anterior, la persona es un ser relacional. Efectivamente, la relación se encuentra en el origen y en el destino de toda existencia humana. El filósofo Martin Buber considera al hombre como un ser dialogal, en continua relación con la realidad $y$, de modo muy particular, con los demás seres humanos, llegando a afirmar: "en el comienzo es la relación" (Buber, 1969, p. 21). El carácter relacional de ser humano no es, pues, entendido desde la simple y lógica necesidad de confluencia de unos con otros en los distintos ámbitos de la vida ni tampoco desde las coordenadas utilitaristas o pragmáticas exigidas por la vida social. Considerar la relacionalidad como característica intrínseca del ser humano supone comprenderlo en un sentido existencial de modo tal que nada que sea propiamente humano puede explicarse completa y auténticamente fuera del hecho irreductible de la relación: "El hombre no puede hacerse enteramente hombre 
mediante su relación consigo mismo sino gracias a su relación con otro" (Buber, 1949, p. 93).

Estas premisas permiten considerar la persona como un ser abierto y relacional, características esenciales que se manifiestan en la unidad de las tres dimensiones que la constituyen: biológica, psicológica y racional (o trascendente - espiritual). En efecto, desde el enfoque de una psicología personalista la intrínseca apertura y relacionalidad humana encuentra sus manifestaciones a nivel biológico, psicológico y racional, siendo posible, a su vez, la síntesis de ambas en la comprensión de la persona completa (Ortiz, Prats y Arolas, 2014).

\subsection{Punto de partida: el cuerpo, símbolo de la persona}

El acceso a la persona nos es posible a través del cuerpo. Tenemos experiencia de que somos más que nuestro cuerpo $\mathrm{y}$, a la vez, no nos explicamos sin nuestro cuerpo.

Una mirada intuitiva a la realidad corporal nos permite atisbar la amplitud de significado que puede albergar, no limitada a la percepción superficial de los atributos externos. El cuerpo tiene la capacidad de revelar, en cierto modo, la interioridad de la persona -mediante el lenguaje, el gesto, el tacto, etc.- y por el mismo medio, la realidad externa puede ser interiorizada entrando a formar parte de la persona de un modo del todo particular, único e irrepetible. Se desprende de ello el valor especial que representa el cuerpo para la persona. Tocando el cuerpo se toca la persona y, a su vez, mediante el cuerpo la persona puede expresarse y entrar en relación con la realidad externa.

El cuerpo, pues, no solo es expresión de un complejo biológico más o menos bien organizado y coordinado -un organismosino que remite a una realidad más profunda, inaccesible a los sentidos la cual expresa de un modo intuitivo; es por ello por lo que afirmamos que el cuerpo es una realidad simbólica: en efecto, el cuerpo es símbolo de la persona y constituye el punto de partida para la propia autoconciencia, autoconcepto y autoestima para el establecimiento de la personalidad, núcleo sólido de la identidad.

¿Qué expresa, pues, el cuerpo? ¿Qué nos dice sobre la persona?

En primer lugar, el cuerpo indica el modo concreto de existencia de la persona humana -como varón o como mujer-. Este hecho queda determinado biológicamente desde las primeras semanas de vida prenatal: la presencia del gen SRY en el brazo corto del cromosoma $Y$ inicia la cadena de microprocesos que conducirán el desarrollo del organismo como masculino $y$, en ausencia de tal gen, el organismo se desarrollará como femenino por la intervención de los distintos factores implicados a partir de la orden cromosómica (López Moratalla, 2012). La cadena de procesos biológicos activados implicará el desarrollo de las gónadas testículos u ovarios- a partir de una gónada indiferenciada que tomará su forma por las órdenes cromosómicas a partir de la $8^{a}$ semana de la gestación; el desarrollo del cerebro -en sentido masculino o femenino, pues el cerebro no es unisex (López 
Moratalla, 2007)-, el desarrollo hormonal testosterona o estrógenos- que intervendrán en la formación de los órganos sexuales internos y externos -fenotipo-. Así pues, biológicamente hay una diferencia esencial entre ser varón y ser mujer empíricamente contrastada (Brizendine, 2007; López Moratalla, 2007), que conduce a funcionamientos diversos de ciertos procesos del organismo -especialmente los cerebrales, por la cantidad de connotaciones derivadas de ello en el pensamiento y el comportamiento-. En efecto, dada la unidad de dimensiones en la persona, lo que sucede en una dimensión presenta sus manifestaciones en las demás, lo cual permite considerar que la diferencia entre varón y mujer alcanza un significado mucho mayor que el estrictamente biológico. Ser varón o mujer no es, por tanto, algo intrascendente, no se trata de un dato baladí: impregna toda la persona de modo completo y definitivo. El cuerpo expresa que somos seres sexuados y cada persona tiene un modo concreto de existencia: como varón o como mujer, que implica todos los niveles del ser personal y así puede decirse que en el ser humano se da un dimorfismo esencial: una forma de realizar la tarea de vivir y habitar el mundo femenina y otra masculina (López Moratalla, 2007).

En segundo lugar, el cuerpo expresa la llamada a la relación intrínseca al ser humano. Hay una huella indeleble en el cuerpo humano que habla por sí misma de la condición de relacionalidad existencial humana: el ombligo. Esta evidencia de nuestra natural vulnerabilidad y necesidad de otro alcanza a la persona completa dotándola de un sentido existencialmente relacional.
Asimismo, los datos anatómicos de los cuerpos expresan que esta relacionalidad presenta una orientación: hacia el otro diferente. $Y$ así, el cuerpo femenino se relaciona con el cuerpo masculino de modo tal que no llega a comprenderse la realidad de uno sin la referencia al otro y viceversa. Así, la condición de relacionalidad entendida como apertura al otro- alcanza su más completa expresión en la relación con el otro totalmente diferente pero semejante a la vez en su condición existencial, reclamándose entre sí en las formas de la acogida y la donación, actitudes básicas en toda relación de unión-amor. El cuerpo, pues, es símbolo de esta natural condición de apertura y relación de la existencia humana, que origina la actitud de búsqueda de unión con otro -amor- con la promesa de alcanzar el sentido a la existencia. Esta necesidad de unión con otro tiene ha de ser comprendida al modo de la persona completa, abarcando todas las dimensiones personales, pues el cuerpo tiene categoría de símbolo -remite a una realidad profunda-, no quedando restringido a los atributos biológicos. Así, la relación varón-mujer es asimismo símbolo de la llamada personal y totalmente única a la apertura en acogida y donación para establecer comunión, esto es, al amor, punto de arranque de toda existencia, así como su destino.

En tercer lugar, el cuerpo expresa que la natural llamada a la relación y unión es de por sí creativa, expansiva, fecunda. La apertura natural a la fecundidad de la unión de los cuerpos femenino y masculino expresa la posibilidad creativa de la comunión interpersonal. 
Con todo, a partir de una mirada atenta al cuerpo como símbolo de la persona, podemos atisbar el sentido existencial de la misma y el camino para alcanzarlo: la llamada totalmente personal vocación- a la relación de unión con otro -el amor- mediante la actitud de acogida y de donación, las cuales generan siempre nuevas posibilidades creativas. El camino de esta vocación al amor comprenderá distintos momentos madurativos, que marcarán las etapas identitarias de la persona desde la experiencia de ser acogido -filiación- a la capacidad de unión -esponsalidad- y de auténtica donación -paternidad-. Podemos comprender así el proceso de configuración de la propia identidad como el desarrollo de la personal vocación existencial al amor, desde un estado inicial de amor hacia sí mismo a partir del amor recibido de otros, que posibilita la apertura al otro y la donación mutua que conduce a la comunión como plenitud de la existencia.

En síntesis, nos situamos ante la persona humana como realidad mistérica, orientada a un horizonte de sentido en el amor que constituye su propia realización existencial. Así, el comienzo de su existencia se entiende en clave relacional, lo cual le procura la orientación de su vida concreta en la unidad de sus dimensiones (necesidad de otros para tener vida y para el desarrollo de las habilidades psíquicas y para alcanzar el sentido existencial). Podemos afirmar, pues, que la relación es connatural a la persona: explica su origen y su destino; una relación orientada por el amor, "la clave de inteligibilidad antropológica por excelencia" (Ortiz, Prats y Arolas, 2014, p. 17).

\section{Identidad personal y sentido existencial. Las paradojas de la identidad}

A diferencia del resto de mamíferos, el humano es el ser animal dotado de una capacidad superior que le permite situarse en la realidad de un modo particularmente proactivo, lo que le permite imprimir en ella su huella. Así, es propio del ser humano cuestionarse acerca de todo lo que acontece en su realidad externa como interna, si bien el mayor interés que presenta es ante las cuestiones relacionadas con su propia existencia. La pregunta por la identidad personal es, pues, connatural al ser humano $y$, a la vez, necesaria para su óptimo desarrollo y realización existencial, pues sin ella no es posible una existencia estable y saludable. Puede tomar diversas formas $\mathrm{y}$ ciertos matices en dependencia de las circunstancias vitales. Si bien esta pregunta se formula normalmente con la expresión: ¿quién soy?, remite a otras similares como: ¿cómo soy?, ¿por qué soy? ¿para qué soy? ¿para quién soy?; cuestiones estas que aluden a diversos aspectos de la propia existencia con las que cada persona trata de comprender y perfilar su propio y particular rostro humano. Preguntarse el hombre por sí mismo implica una introspección no del todo sencilla aun cuando se trata de la realidad de uno mismo; la pregunta por la propia identidad remite, por tanto, a una realidad que trasciende a las propias características fácilmente accesibles. La pregunta por la identidad personal es la pregunta por el sentido existencial.

Con todo, el estudio sobre la identidad personal requiere una comprensión clara de a lo que nos estamos 
refiriendo. ¿En qué consiste, pues, la identidad?

El Diccionario de la Real Academia Española (R.A.E., 2019) indica que "identidad" proviene del latín tardío identǐ tas, y este derivado del latín idem 'el mismo', 'lo mismo'. Entre los significados ofrecidos, destacamos el segundo y el tercero descritos por hacer referencia al ámbito que nos ocupa:

(2) conjunto de rasgos propios de un individuo o de una colectividad que los caracterizan frente a los demás;

(3) conciencia que una persona o colectividad tiene de ser ella misma y distinta a las demás.

Si bien la definición pudiera parecer clara a primera vista, no lo es tanto en el momento en que se trata de aplicar a la propia realidad personal. ¿Qué podemos considerar como rasgos propios? ¿En qué consiste la conciencia de ser uno mismo, dados los continuos cambios que se van produciendo en la historia personal? ¿En qué radica, esencialmente, la diferencia con los demás? ¿No se da, asimismo, una clara semejanza entre todos los seres humanos?

Efectivamente, encontramos que somos semejantes y diferentes a la vez y que la vida personal está sometida al continuo cambio. Así, todos tenemos experiencia de los cambios que se van produciendo a lo largo de nuestro desarrollo temporal, descritos con evidencia científica por la Psicología del Desarrollo. Asimismo, tenemos experiencia de que en nosotros se suceden cambios que no tienen causa temporal, sino que se deben a otros elementos tales como el ambiente socio-cultural, las relaciones interpersonales, etc. $Y$ también tenemos la evidencia de los cambios que nosotros mismos podemos originar mediante el ejercicio de nuestra libertad. Entonces, ien qué consiste, exactamente, nuestra identidad? Nos encontramos con varias paradojas en la comprensión de esta realidad que nos disponemos a considerar:

\subsection{Singularidad y homogeneidad}

Por una parte, tener identidad consiste en tener conciencia de la propia individualidad, comprender la propia existencia como única e irrepetible $y$, al mismo tiempo, tener conciencia de la homogeneidad con los semejantes, esto es, la conciencia de formar parte de una colectividad.

Así, la identidad exige y se explica por la relación con los demás, elemento esencial de la propia identidad, ante quienes uno ha de mostrarse como sí mismo diferenciado a la vez que como parte de la totalidad, si bien al modo personal, esto es, considerando su unicidad e irrepetibilidad, su inalienable dignidad.

En un sentido estrictamente biológico, podemos considerar que los rasgos de cada persona se deben, en gran medida, al factor hereditario sobre el que, desde el inicio de la vida, los procesos epigenéticos actúan dando lugar a los rasgos propios de cada individuo. Esto nos lleva a afirmar, por tanto, que la identidad personal asume en sí misma la diferencia y la igualdad en sentido ontológico en una perspectiva relacional. 


\subsection{Intersubjetividad e intrasubjetividad; interioridad y exterioridad}

La conciencia de ser uno mismo y distinto a los demás requerirá, asimismo, desarrollar el propio conocimiento en dos aspectos: por una parte, comprenderse a sí mismo y tener experiencia de la propia interioridad -el ámbito de la intencionalidad y motivación, tendencias e íntimas inclinaciones-, tratar de ir desvelándose el propio misterio que la misma persona es para sí; por otra parte, comprenderse a sí mismo en continuo diálogo con la realidad circundante, en la que se pone en juego la propia interioridad implicándose en la relación, a la vez que la relación deja huella en la propia interioridad de la persona.

\subsection{Estabilidad y cambio}

La identidad personal supone la conciencia de permanencia ante los diversos y continuos cambios que se producen de forma inevitable en el devenir de la vida. Se trata entonces de identificar aquel núcleo invariable, lo que resiste a los cambios biográficos (Polaino Lorente, 2008).

Tener identidad consiste en la conciencia de que no somos los mismos pues estamos en continuo cambio, un proceso ininterrumpido de cambios de diversa índole, de modo gradual- pero, a la vez, tenemos la seguridad de que somos los mismos a través de los periodos o cambios en cada uno de los momentos y etapas de mi desarrollo, soy yo mismo-. Los cambios son solo accidentales, son atributos que no modifican el núcleo estable del sí mismo.

\subsection{Naturaleza y libertad}

Otra paradoja de la identidad personal lo constituye la realidad de que es "en cierto modo lo dado y en cierto modo lo alcanzado" (Polaino Lorente, 2008, p. 117). La identidad supone tener conciencia de que la propia existencia se sostiene en una base lo dado- sobre la cual puedo actuar y me voy haciendo, pues la persona es, pero no está hecha. Esta dualidad ha generado controversias de no poca importancia por las consecuencias que se han derivado de las distintas interpretaciones ofrecidas acerca de la interrelación de estas realidades humanas.

No obstante, otras aportaciones revelan de modo clarificador la síntesis entre los dos elementos de este binomio intrínseco a la existencia humana. En este sentido podemos destacar como sumamente clarificadora la siguiente afirmación del filósofo contemporáneo Antonio Millán Puelles: "somos libres, o sea, no estamos hechos del todo; pero somos, o sea, no todo lo tenemos que hacer" (1974, p. 304). Esta comprensión de la realidad humana en la sencillez y realismo de su argumentación nos sitúa ante el horizonte de sentido existencial de la vida humana: no somos creadores de nuestra existencia, pero podemos intervenir en la realización de nuestro destino.

En síntesis y consecuencia de todo lo anterior, podemos establecer una última paradoja o dualidad: la identidad personal supone a la vez un don y una tarea. La identidad, pues, no es algo que nos viene 
"dado" sino que es un logro, se ha de conseguir mediante una tarea que toda persona ha de asumir de modo ineludible e insustituible, pues en ello está implicado su sentido existencial.

Esta tarea personal es descrita por M.L. di Pietro (2013, p. 265) de la siguiente manera, señalando los elementos necesarios:

Una de las grandes "tareas" con las que toda persona debe confrontarse durante su crecimiento es la estructuración de la propia identidad, es decir, la capacidad de autorrepresentarse y percibirse como sujeto unitario con características y cualidades estables, permanentes y distintas de las de los demás. El desarrollo de tal capacidad requiere un camino largo y gradual para adquirir conciencia y conocimiento de sí mismo.

Finalmente, una visión equilibrada del proceso de configuración de la identidad personal supone considerar la interdependencia del conjunto de facultades, rasgos y habilidades con las que la persona interactúa en la realidad incidiendo sobre su propia naturaleza y que la van configurando en un sentido del todo originario y único. Así pues, también toma parte en la propia identidad aquello a lo que la persona se orienta -sus amores, las relaciones que establece con la realidad- (Polaino Lorente, 2010). Finalmente, siguiendo las evidencias científicas desde el ámbito de la psicoterapia, podemos concluir que la identidad es esencial para el desarrollo de una vida lograda y el cumplimiento del sentido existencial: "la identidad es lo que de verdad alienta en la persona. Una persona sin identidad se ha quedado sin voz para a sí misma llamarse, hacerse presente, conocerse en su singularidad y manifestares tal cual es" (Polaino Lorente, 2008, p. 121).

Desde la perspectiva descrita, se ha considerado la identidad personal en clave relacional desde diversos aspectos. Así pues, el propósito de las siguientes líneas es argumentar el aporte esencial del factor interpersonal en el proceso de la configuración de la identidad.

\section{El factor interpersonal en la configuración de la identidad}

La configuración de la propia identidad supone una conquista larga y progresiva. La identidad ya le viene dada a la persona en el primer momento (Polaino Lorente, 2008) en germen, que ha de ser expresada y plenificada a lo largo de la existencia. El proceso en sí comienza con el inicio de la propia vida, esto es, en el momento de la fecundación, si bien el aspecto más concreto referido a la conciencia de sí mismo tendrá sus primeras evidencias hacia los dos años. La infancia constituye una etapa relevante en este desarrollo y, alcanzando la adolescencia exigirá una especial atención por los factores implicados y las diversas perspectivas que se presentan ante la persona. Aunque podemos considerar, sin lugar a duda, que la identidad se desarrolla a lo largo de todo el proceso vital, es preciso sin embargo destacar la importancia de las etapas de la infancia y la adolescencia por los notables progresos evolutivos que en ellas se suceden y que sirven de soporte de la identidad. 
La investigación en psicología de la personalidad destaca el aporte particular de la relación interpersonal en la adquisición de las habilidades psíquicas requeridas para la configuración de una existencia estable y saludable. Tal como sostiene Julián Marías (1995), el yo de cada persona está instalado socialmente, y así no es posible considerar su desarrollo desligado del entramado de relaciones interpersonales en que se ve involucrado. La memoria del propio origen y de la propia trayectoria vital sirve de suelo firme de la identidad (Polaino Lorente, 2010), lo que se consigue a través de las experiencias tempranas de relación interpersonal. Estas van formando la memoria biográfica, el "marco conceptual desde el cual el niño entiende el mundo y se comprende a sí mismo" (L'Ecuyer, 2015, p. 101). A partir de esas primeras relaciones interpersonales y de las sucesivas que mantendrá con las personas significativas en cada etapa madurativa, irá adquiriendo la imagen del propio yo -la identidad- y sus correspondientes contenidos de conciencia, dando lugar progresivamente a actos regulares y estables (de Gregorio, 2007).

Para guiar el estudio sobre la importancia de la interacción personal en la configuración de la identidad, seguiremos las etapas establecidas en la teoría científica elaborada por el psicoanalista Erik Erikson sobre el desarrollo psico-social, por las claras pistas orientativas que ofrece a nuestro interés.

Erikson (2000) interpreta el desarrollo de la conciencia personal mediante la interacción social y así, establece el desarrollo psico-social a lo largo de una secuencia de etapas en las que la persona se enfrenta a distintos retos madurativos. La superación exitosa de cada etapa supone la adquisición de una habilidad psico-social y el avance en la conciencia de sí o identidad. Asimismo, en cada etapa está implicada una particular relación interpersonal, a través de la cual la persona pone en juego sus habilidades para conseguir el logro de una nueva adquisición madurativa. Así pues, la personalidad se va desarrollando a través de los contextos específicos y las relaciones interpersonales que, en cada momento existencial, se van sucediendo. Cada etapa supone, según Erikson, un conflicto o crisis, esto es, un momento de crecimiento, en el cual se produce un cambio en el modo de percibir la realidad y relacionarse con ella según las distintas necesidades y expectativas ante las situaciones que presentan nuevas cotas de desarrollo o retos madurativos. El desarrollo individual se va adquiriendo, así, mediante la superación de los conflictos o crisis madurativas. A diferencia de otras conocidas teorías evolutivas de la personalidad que establecen su desarrollo hasta el momento de la adolescencia, Erikson lo comprende durante todo el ciclo vital. Así, entiende la vida como un sucederse de conflictos en los que la persona pone en juego sus habilidades y manifiesta su potencialidad de desarrollo, resultando estancado cuando no encuentra los recursos necesarios para su resolución exitosa. Del éxito o fracaso de cada etapa madurativa dependerá la configuración de la propia identidad personal.

Desde este marco interpretativo, podemos dar inicio al estudio de la interacción personal como recurso esencial y específico para cada etapa del desarrollo de la propia identidad. En las siguientes líneas 
nos ocuparemos de especificar las relaciones interpersonales más involucradas en cada etapa evolutiva y las capacidades que favorece alcanzar. Las distintas etapas madurativas pueden ser comprendidas como un progreso en la capacidad de relación interpersonal, derivado de la progresiva adquisición de las habilidades personales.

\subsection{La etapa prenatal}

El punto de arranque hacia la conquista de la conciencia de sí mismo lo constituye la evidencia de que nadie llega a este mundo fuera de una relación originaria: la relación familiar, fundada en la relación de dos diferentes entre sí (unión del ADN de la madre y el ADN del padre constituyendo un ADN nuevo, único e irrepetible). Se establece de este modo el primer núcleo relacional, fundante de la propia vida humana, y dado el carácter de símbolo de la realidad corporal, esta relación fundante será igualmente esencial para el desarrollo psíquico y existencial de la nueva vida humana originada. La realidad del ser humano supone no poder encontrarse en la existencia fuera de la relación: una relación que le precede y que le da origen y sin la cual no puede lograr una comprensión apropiada de su trayectoria existencial. La relación, pues, forma parte de la realidad mistérica de la persona: cada una, remite a la relación entre las dos personas de las que proviene. Importante, pues, esta relación de diferentes no solo para el origen sino para toda la trayectoria del camino existencial.

La etapa prenatal no constituye, por tanto, un tiempo en off para el desarrollo de la identidad del hijo como se pudiera de algún modo pensar. La relación en la que, desde el inicio de su vida, se encuentra el nuevo ser humano y que se irá haciendo consciente a medida que avance el desarrollo de sus órganos y capacidades, ejerce una notable influencia para el desarrollo incipiente de su personalidad $y$, por lo tanto, de su identidad. De modo particular la relación se dará con la madre, dada la unión biológica, si bien la relación con el padre será igualmente importante, aportando otros elementos para el desarrollo y percepción del mundo por parte del hijo. La importancia que tiene esta relación del hijo con el padre y con la madre durante la etapa gestacional ha sido destacada mediante la denominación de "triangulación originaria" (Tizón, 2016, p. 7).

Como ha evidenciado la literatura científica, la gestación constituye un periodo sumamente significativo para el establecimiento del vínculo madre-hijo, originado a nivel biológico por el delicado diálogo molecular que establece el organismo del hijo con el de la madre en los primeros instantes de modo inconsciente para la mujer por el cual el hijo es acogido por la madre mediante las hormonas liberadas por ambos (López Moratalla, 2008). Se evidencia que el organismo femenino está dispuesto naturalmente a la acogida de la vida humana, estableciéndose así la primera relación interpersonal del ser humano: con su madre. Una vez que la madre tenga certeza del embarazo podrá desarrollar afecto positivo, lo que potenciará ese primer lazo biológico ya establecido y adquirirá auténtica conciencia de la relación generada con el hijo, lo que tendrá sus implicaciones en el propio desarrollo del hijo. La investigación científica ya cuenta con 
numerosas evidencias sobre las consecuencias del vínculo afectivo prenatal para el hijo y para la madre, así como las características de esta primera relación interpersonal y se sostiene su influencia en el desarrollo incipiente de la personalidad y de ciertas aptitudes (Verny, 2011; Fernández Mateos, 2005). Tan esencial puede resultar esta primera relación interpersonal que mantenemos en el propio cuerpo el recuerdo indeleble de la misma: el ombligo. A medida que la persona avance en su desarrollo, la memoria del origen, de la primera relación fundante y de la primera relación interpersonal, será un dato esencial para el desarrollo de la propia conciencia de sí (Polaino Lorente, 2010).

Durante la etapa prenatal se produce el desarrollo de los principales tejidos y órganos según las indicaciones genéticas en sentido masculino o femenino y en el momento del nacimiento ya estarán formados los distintos órganos sexuales y genitales internos y externos. Esto será necesario para el proceso de autopercepción que tendrá lugar en los primeros años después del nacimiento. Si en algún modo se produjera cierta alteración en alguno de los procesos implicados en el desarrollo sexuado originando anomalías en los órganos sexuales externos o internos -casos de indefinición sexual, de bajísima incidencia llamados intersexos- se debería considerar con la debida prudencia y respeto a la dignidad de la persona al tratarse de un aspecto tan relevante para el propio desarrollo e identidad. Así, la línea de intervención en estos casos vendría dada por la adecuación de los genitales a la información genética, núcleo sólido de la identidad (Aznar y Tudela, 2017).

\subsection{Etapa neonatal}

Según establece Erikson (2000), durante los primeros 18 meses de vida extrauterina, el recién nacido se encuentra en el conflicto entre la confianza y la desconfianza. La persona llega a este mundo en el contexto de una relación personal y como receptividad relacional; su apertura a la realidad es pasividad relacional con otra persona (Domínguez, 2007). El recién nacido está capacitado para abrirse a la realidad en actitud de encuentro mediante la habilidad para la comunicación que irá madurando desde la forma más básica del llanto y las muestras de agrado que serán sus primeras estrategias de relación. Mediante los cuidados que reciba, podrá tener la sensación de protección y seguridad en que va a sobrevivir a las amenazas del mundo nuevo en el que ha empezado a vivir, o, por el contrario, experimentará la inseguridad y frustración al verse amenazada su supervivencia. La capacidad madurativa de la esperanza, resultante de la vivencia positiva de esta etapa, se acompañará de un amplio abanico de sensaciones y afectos positivos que permitirán la actitud de apertura del recién nacido a la realidad que le rodea, formada en ese momento por el núcleo familiar más estrecho formado por los padres. De lo contrario, la experiencia del desamparo le provocará afectos negativos expresados como angustia en la relación.

En esta etapa la figura principal de relación sigue siendo la madre, cuidador principal, como soporte del vínculo afectivo. La adquisición de la confianza básica o apego, cristalizado entre los 12 y los 18 
meses, está mediado fundamentalmente por el contacto físico, a partir de los primeros cuidados básicos de alimentación e higiene, quedando ligado a la supervivencia. Su importancia es tal que su ausencia en los primeros momentos de la vida extrauterina se asocia con notables carencias madurativas y desequilibrios psico-afectivos (Bowbly, 2006; Montagú, 2004).

No obstante, la madre deberá hacer espacio para el que recién nacido tome relación directa con el padre, cuyo papel será destacado en esta etapa por posibilitar al neonato la experiencia de la realidad completa en su dualidad y la apertura a la sociabilidad y al mundo de las relaciones sociales. El padre constituye "la otra figura de apego" (Quaglia y Vicente, 2007, p. 169) cualitativamente diversa a la madre $y$ totalmente necesaria para el desarrollo del hijo. De modo particular, su importancia es decisiva por intervenir y favorecer el proceso de separación necesaria de la fusión madrehijo en la que se encuentra al llegar a este mundo, pues sigue de algún modo viviendo aquella relación de total unión con la madre que se dio durante la gestación. Pero vivir en este mundo requiere adquirir conciencia de ser uno mismo, para lo que tendrá que romper el cordón umbilical afectivo que los mantiene fusionados para permitir la adquisición de la conciencia de sí como una individualidad, separado del cuerpo de la madre, lo que le permitirá abrirse a la percepción de la realidad de un modo independiente. La apertura a la relación con una tercera persona especialmente cercana la figura del padre- resulta del todo esencial para el proceso de individuación del hijo y para el establecimiento de las pautas y la regulación de la satisfacción, elemento necesario para el equilibrio de la personalidad. El padre, pues, supone la figura de orden: para la madre, al favorecer la separación del hijo por momentos que ella misma debiera propiciar para que el padre pueda entrar en relación con el hijo, y para el hijo, pues le permite la experiencia de los tiempos en la realidad y así hay un tiempo para la satisfacción de necesidades algunas de ellas especialmente vinculadas a la madre $\mathrm{y}$ otros tiempos para la actividad e interacción en las que entra en juego especialmente la figura del padre y otras figuras distintas del entorno cercano. En la dinámica de estas primeras relaciones particularmente en la relación triangular padre-madre-hijo- la identidad va adquiriendo los elementos que servirán de soporte básico para su completo y complejo desarrollo posterior.

\subsection{Primera infancia}

Hacia los dos años, aparecen los primeros atisbos de autoconciencia en el niño -se puede reconocer ante un espejo(Vasta, Haith y Miller, 2001, p. 576) y empieza a elaborar el autoconcepto (la idea de sí mismo) y la autoestima (el afecto hacia uno mismo), elementos clave de la propia identidad.

La adquisición de las nuevas habilidades motrices -locomoción, habilidad manual- y simbólicas -lenguaje, dibujo, juego-, permiten ampliar y confirmar la experiencia de individualidad y de control sobre su propio cuerpo que el neonato ha ido alcanzando en las primeras relaciones interpersonales y le capacitan para dar una respuesta más individualizada que aquellas 
primeras reacciones espontáneas de la etapa anterior. Se le abren así al niño nuevas oportunidades para explorar el mundo, lo que ampliará las posibilidades de su autoconocimiento, limitado hasta ese momento a su propio contacto corporal. Erikson (2000) considera que el niño se enfrenta en este momento (18 meses-3 años) a la necesidad de equilibrio entre la autonomía y la vergüenza. La capacidad de voluntad incipiente en el niño deberá encontrar un óptimo escenario, dentro de la razón y los límites apropiados, para poder desarrollarse y superar con éxito esta etapa madurativa. De lo contrario, el niño experimentará vergüenza y duda ante sus nuevas habilidades menoscabando su apertura a la realidad y dando lugar a actitudes de retraimiento de sí mismo ante las nuevas relaciones.

A partir de la interacción con las demás personas de su entorno más cercano, el niño irá configurando una idea de sí mismo y de su propia capacidad y valía. Así, será fundamental para este proceso el tipo de mensajes que reciba de las personas más cercanas, las palabras y los tonos que utilizan al dirigirse a él, la forma de atenderle y de interactuar. El niño irá de este modo construyendo una idea de sí mismo que desencadenará una serie de actitudes y de afectos que marcarán su pensamiento y sentimientos. En efecto, "el niño aprende a comprender el "sentido" o valor de sus propias acciones, comprendiendo el "sentido" o valor que a las mismas les dan aquellos con los que se relaciona" (de Gregorio, 2007, p. 370). Todo ello contribuye de modo esencial a la gestación del núcleo de la autoestima, que en estos momentos se inicia y que constituirá aquel centro profundo y estable del afecto hacia sí mismo, generado a partir del afecto que percibe y recoge de los demás. La propia identidad se asienta sobre la experiencia de este afecto básico, destacando de nuevo la clave relacional.

La interdependencia de los demás es, pues, el eje fundamental durante los primeros años para la percepción de la realidad y de sí mismo. En los años sucesivos esta interdependencia seguirá teniendo importancia si bien tomará diversos matices a tenor de las capacidades madurativas que irá adquiriendo la persona en su proceso de desarrollo.

La capacidad de autopercepción adquiere mayor amplitud cuando el niño empieza a mostrar curiosidad por su propio cuerpo que desde el principio ha tomado como fuente de satisfacción e interacción. El niño comienza a autoexplorarse conscientemente y descubre nuevas sensaciones placenteras localizadas en zonas concretas $y$, según el criterio básico de su comportamiento, tenderá a buscar esa satisfacción. Su curiosidad le orienta a buscar y captar las diferencias entre los diversos cuerpos y órganos sexuales y así puede ser característico observar a niños y niñas explorándose unos a otros las distintas zonas genitales a la vez que establecen sus argumentos explicativos y formulan a los adultos sus curiosidades al respecto. El modo como se interpreten y traten estos comportamientos por parte de los adultos cercanos al niño tendrá notables implicaciones en la interpretación que el niño vaya elaborando acerca de su cuerpo y las nuevas características que está descubriendo. Así también, el modo en que 
sean recibidas y respondidas las preguntas de los niños sobre sus nuevos descubrimientos corporales irán orientando la percepción de sí mismo como alguien valioso o, por el contrario, se sentirá negado, rechazado o no considerado importante, generando sentimientos negativos hacia sí mismo. Se trata de un momento de conocimiento que requiere ser comprendido de un modo equilibrado y realista para fomentar el desarrollo óptimo del infante. De nuevo la relación interpersonal, en este caso de los padres o cuidadores cercanos, cobra valor destacado como canal de interpretación de la propia realidad sexual que el niño está comenzando a percibir y experimentar, así como de las diferencias entre sexos.

Cuando ya está avanzada la autopercepción, entre los 3 y 5 años, Erikson (2000) establece una nueva etapa conflictiva: iniciativa-culpa, que provocará la capacidad de propósito. Los progresos cognitivos permiten al niño aportar su iniciativa en las acciones cotidianas que observa a su alrededor: experimenta cierta creatividad y capacidad de establecer propósito en sus acciones, como consecuencia de la autonomía y voluntad logradas en la etapa anterior. Así, el infante se considera partícipe en la vida social de su reducido entorno pudiendo realizar su aportación particular, lo que le proporcionará una experiencia previa y necesaria de los patrones que rigen la vida en sociedad.

Una especial importancia tendrá en este proceso la figura del padre del mismo sexo a fin de favorecer la identificación que haga posible formular en su pensamiento la realidad simbólica de lo que significa ser niño o niña. Supone este un momento esencial y delicado en la trayectoria de la propia identidad que requerirá el contacto de calidad con el padre del mismo sexo -o referente sexual apropiado- mediante el juego, la conversación, la actividad conjunta, etc. (Polaino Lorente, 2007). Debido al desarrollo cognitivo, la capacidad de pensamiento simbólico le permite la imitación de modelos significativos de su entorno. La interacción con el padre del mismo sexo tendrá asimismo efecto modelador de ciertas actitudes y conductas por el aprendizaje social cognitivo (Bandura, 1982); el niño interiorizará aquellos comportamientos o actitudes que vea en su referente, de modo tanto más significativo cuanto más fuerte sea el vínculo afectivo entre ambos, conformando así un modo de pensar y de sentir hacia la realidad de ser mujer o varón, que se expresará en su conducta.

Un momento de particular relevancia consiste en la aparición espontánea del sentido del pudor a los 3- 4 años: un sentimiento natural de protección ante la conciencia cada vez más creciente, de ser alguien único y poseer una intimidad. Una vez que el niño ha hecho sus avances en la autopercepción y conciencia de sí, así como en la diferencia entre las personas, tomará conciencia de la propia realidad interior, su intimidad y experimentará la necesidad de reservarla ante los otros. Es un momento especialmente delicado que requiere ser comprendido y adecuadamente acompañado y orientado para que no se produzcan interpretaciones falseadas del avance madurativo que supone: el pudor no es represión ni vergüenza. Algunas conductas frecuentes que manifiestan este 
avance pueden ser que el niño comience a guardar sus cosas con cierto cuidado y que incluso esconda aquellas que le son más significativas y las considere "secretas", que empiece a cerrar la puerta de su cuarto al vestirse y la del aseo. El comportamiento se hace más autónomo, exige cierta independencia en sus actos con respecto a sus cuidadores. Comprender este proceso como una nueva adquisición y no como un comportamiento represivo es fundamental para favorecer el desarrollo del autoconcepto y autoimagen, elementos esenciales para la propia identidad. Se hace especialmente importante en este momento que niño y niña tengan una especial interacción con el padre y la madre respectivamente, favoreciendo el cuidado y el respeto por la propia intimidad del niño según las señales que vaya dando. El sentido del pudor puede verse dañado si el niño recibe mensajes de desinterés hacia esta nueva adquisición y ve menospreciados sus comportamientos incipientes referidos al cuidado corporal y hacia sus cosas personales. Un tratamiento equilibrado de este hito evolutivo favorecerá que el niño valore su propio cuerpo como expresión de su realidad interior y no como simple exterioridad, lo que conducirá a reservarlo de un uso superficial y disponer plenamente de él para que pueda expresar la donación personal auténtica. El sentido del pudor supone, pues, el germen de la virtud de la castidad, como señala David Isaacs (1986, p. 197): "ayuda a la persona a autoposeerse en algún grado para luego entregarse en el momento oportuno". Por todas las consecuencias que se derivan de ello, las relaciones interpersonales implicadas en esta etapa evolutiva resultan especialmente significativas para la configuración de la propia identidad.

\subsection{Segunda infancia}

Quizá lo más característico en esta etapa sea la exigencia de socialización fuera del ámbito familiar, en el que ha tenido la experiencia de la acogida $y$, por otra parte, ha aprendido y desarrollado las pautas esenciales de la interacción social mediante las primeras relaciones interpersonales. Asimismo, las nuevas habilidades cognitivas alcanzadas por el mayor grado de madurez del cerebro le permiten empezar a manejar abstracciones $y$, poniendo a prueba su capacidad de voluntad y de propósito, busca obtener reconocimiento a sus tareas.

En la interacción con los iguales, aparecen en el niño nuevas necesidades: expresar las propias capacidades para hacerse valer y ser reconocido capaz. Serán frecuentes, pues, en este momento, las comparaciones con los iguales y el consiguiente establecimiento de categorías (el más divertido, el más rápido, el más listo, etc.). Erikson (2000) formula en esta etapa ( 5 a 13 años) el conflicto entre laboriosidad $e$ inferioridad, y la adquisición de la capacidad madurativa de competencia. Así, el niño que va asumiendo nuevas tareas normalmente asociadas al entorno escolar, se enfrenta al reto de lograr sentido de capacidad personal o, de lo contrario, de inferioridad, lo que se verá influido por la apreciación que su entorno haga de sus propias habilidades. Un entorno favorable a la comprensión y valoración de las capacidades del niño sin sentido de rivalidad, sino desde la apreciación de su realidad y el 
fortalecimiento y desarrollo de sus potencialidades, será el más apropiado para la percepción equilibrada de las relaciones interpersonales que suponen el escenario en el que el niño comienza a mostrarse a los demás. Una percepción equilibrada de sí mismo le orientará a mantener relaciones basadas en la mutua interrelación; sin embargo, una infravaloración de sí le llevará a buscar contextos de seguridad en los que no se evidencie la incapacidad que experimenta.

Esta etapa constituye también, por los avances y retos descritos, un momento apropiado para la comprensión de las cualidades propias de lo femenino y de lo masculino mediante una visión positiva e integradora de ambas realidades sexuales, para lo que será preciso prescindir de la interpretación dualista en términos de superioridad o inferioridad. Para ello, el niño requerirá la presencia en su entorno cercano de personas con vinculación afectiva que sean referentes de lo específico masculino y lo específico femenino y transmitan de un modo espontáneo la experiencia de la relación armoniosa entre los sexos. Elemento crucial para la configuración de la identidad sexual es la percepción y comprensión de que las diferencias descubiertas en los cuerpos tienen un sentido y cada tipo de persona -masculina y femenina- es valiosa en sí misma y presenta unas características particulares e intercambiables necesarias para la vida en relación y la construcción de la sociedad. Sobre esta cuestión es de destacar la notable influencia de los modelos ofrecidos en las redes sociales y medios de comunicación a los que el niño de estas edades ya suele tener acceso con cierta independencia del control adulto. Así también merece particular interés en esta edad el estilo de ocio del niño: un tiempo de ocio creativo y positivo establecerá relaciones con otros niños o adultos en contextos constructivos mientras que un ocio pasivo podrá derivar en incipientes conductas adictivas mediadas por lo general por los medios tecnológicos disponibles, generando actitudes de aislamiento o a la notable reducción de las relaciones interpersonales.

\subsection{Adolescencia}

Erikson (2000) sitúa en esta etapa (1321 años) la crisis existencial fundamental identidad-confusión que proporcionará el logro de la capacidad madurativa de la fidelidad. Se trata de un momento crucial para el establecimiento de la propia identidad, comprendida como el conocimiento y conciencia de sí, si bien, como ya quedó argumentado, todo el ciclo vital es para Erikson un camino evolutivo de la propia conciencia de sí.

El hecho que marca la entrada en esta etapa es la irrupción de la pubertad como proceso biológico: cambios bruscos en la producción hormonal generan cambios destacados en el aspecto físico. Se produce un desequilibrio entre el desarrollo biológico -que ha alcanzado una nueva cota: la capacidad fértil, y con ella la entrada a nuevas realidades existenciales- y el desarrollo psíquico -destacada inmadurez cerebral al no haberse alcanzado aun la maduración del lóbulo frontal, centro coordinador del control de impulsos-. El adolescente se encuentra, de repente, en un mundo totalmente desconocido para él y sin 
poseer los recursos para gestionar los numerosos cambios que se van produciendo en su realidad vital, lo que presenta, normalmente, la forma de confusión en la identidad y ello explica el comportamiento y pensamiento del adolescente.

Así, el niño que se convierte en adolescente mediante la pubertad se enfrenta a un nuevo reto: consolidar su identidad ante los nuevos cambios que está experimentando. Todo el conocimiento de sí mismo que ha ido adquiriendo en las etapas anteriores mediadas por las relaciones interpersonales que ha ido estableciendo, se pone a prueba en la adolescencia. La nueva exigencia que se le presenta es adquirir convicciones profundas, certezas sobre las que construir su proyecto existencial y para ello, todo aparece ante él como dudoso. Por un lado, exige mayores niveles de independencia, por lo que se desvincula en cierto modo de los patrones de conducta y criterios mantenidos en el ámbito familiar o entorno más cercano. Los progresos cognitivos le exigen buscar las razones lógicas a los hechos, a las ideas que ha ido formando sobre el mundo, las cosas, las relaciones personales, etc., aunque reduce su centro de interés y preferencia a su entorno afectivo de iguales, en el que se siente aceptado, valorado y comprendido, respuestas a su necesidad de pertenencia. Debido a la desorientación general que experimenta en distintos ámbitos de su vida, a la exigencia de independencia se une la necesidad de apoyo constante para tomar decisiones y establecer los criterios del propio comportamiento y para el juicio moral de la realidad.
La superación exitosa de esta etapa supondrá el ejercicio de conocimiento real de sí mismo, la aceptación de su propia realidad y la comprensión de su valor, de modo que se vea estimulado a abrirse al futuro de un modo constructivo, con capacidad para realizar su aportación personal de modo consecuente. Es el momento, normalmente, de la elección de estudios superiores $y$, quizá de adquirir cierta independencia del seno familiar e incluso de iguales. Todo ello requiere, en cierto modo, haber alcanzado ciertas cotas en la definición de la propia identidad, que le permitan actuar con coherencia personal o fidelidad a sí mismo. Si, por el contrario, no se consigue tal conocimiento de sí, la persona quedará estancada en un estado de inmadurez y confusión en la identidad, que le impedirá abrirse al futuro de un modo equilibrado y constructivo en actitud de acogida y donación, pudiendo desembocar incluso en patologías psíquicas.

De modo habitual se establece que la identidad queda configurada en la adolescencia, si bien de un modo aun voluble, que requerirá avanzar en su cristalización en las etapas posteriores.

\subsection{Adultez temprana}

Los avances en las etapas anteriores han ido procurando la adquisición de las capacidades apropiadas que permiten al joven en este momento contemplar la llamada al encuentro interpersonal, a la comunión. Así, Erikson (2000) plantea la etapa intimidad-aislamiento (21-39 años) para poder alcanzar la capacidad del amor. Una vez establecida básicamente la 
identidad, la persona se ve capacitada para orientarse a relaciones interpersonales íntimas y recíprocas, en las que se manifiesta su existencial disposición a la acogida y la donación. Este avance marca el paso a una nueva etapa identitaria: la esponsalidad: la disposición y capacidad para el encuentro interpersonal y de construir un vínculo de comunión, que, de por sí, presenta potencialidad creativa y difusiva.

\subsection{Adultez media}

La potencialidad difusiva de la comunión -el encuentro completo $y$ definitivo en acogida y donación- da paso a una nueva etapa identitaria: la paternidad. Como afirma Scola (2002, p.116): "para ser padre se requiere seguir siendo hijo", esto es, reconocer su condición filial pues, en efecto, nadie estamos en la existencia fuera de ella, marcando de modo definitivo la identidad. Así, la trayectoria de la vocación al amor desde la acogida a la donación personal se sostiene sobre la experiencia fundamental de saberse acogido y amado, que capacita para el encuentro interpersonal y la comunión creativa.

Erikson (2000) establece en esta etapa la dualidad generatividad-estancamiento, a través de la cual la persona ha de alcanzar la capacidad del cuidado de otros. De este modo, su vida se verá realizada y plenificada en la actividad creadora -pudiendo ser entendida en los distintos ámbitos existenciales, no solo en el biológicosiempre en la acogida y donación a otros, esto es, en actitud amorosa. Siguiendo esta trayectoria, la persona será capaz de cumplir con éxito la siguiente etapa integridad- desesperación, que sitúa a la persona en la oportunidad de desarrollar la virtud de auténtica sabiduría, la cual le permitirá contemplar la propia vida de modo integrador. Así, la persona verá realizado su sentido existencial en la vida de comunión y alcanzará felicidad por la vida lograda.

\section{Conclusiones}

- Al llegar al término de este trabajo, exponemos a continuación las conclusiones más relevantes en relación con la tesis planteada al inicio: la vinculación entre relacionalidad e identidad personal y la consiguiente comprensión del sentido vital como vocación al amor.

- En primer lugar, partiendo de las dos claves interpretativas de la persona apertura y relacionalidad- se ha afirmado que la vida humana está orientada al encuentro con otro, lo cual le confiere identidad y realización personal. Se ha considerado que el cuerpo presenta categoría de símbolo de la persona, en cuanto realidad sensible que evoca una realidad no accesible a los sentidos. Asimismo, es la vía de acceso a la persona y su medio de expresión, elemento por tanto esencial de la identidad y propia realización humana.

- En segundo lugar, la reflexión realizada sobre el proceso de la configuración de la propia identidad permite sostener que éste implica la síntesis entre las diversas dicotomías de la realidad personal: diferencia y 
unicidad, don y libertad, cambio y permanencia, dependencia y autonomía, etc. Estos prismas de la identidad se conjugan de modo misterioso, como misteriosa es la persona, que remite siempre a un nivel superior para lograr su comprensión. Así, la identidad se configura en un proceso dinámico y creativo totalmente único que tiene lugar durante toda la vida, tomando especial relevancia en la etapa de la infancia y la adolescencia.

- En tercer lugar, se ha considerado que el desarrollo humano está orientado a capacitar a la persona para la interacción con los demás. A su vez, se ha destacado el papel fundamental de la relación interpersonal en el proceso de desarrollo de la propia identidad personal y sentido vital. Esto se ha descrito mediante el recorrido por las etapas evolutivas del desarrollo psico-social propuestas por Erikson, mostrando la implicación de relaciones interpersonales significativas en cada una de las etapas psicosociales y evidenciando su papel fundamental para la resolución de los conflictos madurativos presentados en cada una de ellas. Desde la primera relación con la madre, la persona se encuentra en un entorno social -relacional- que supone el punto de arranque y desarrollo de las propias habilidades psíquicas. En el seno de las primeras relaciones interpersonales en el entorno familiar, ligadas al cuidado y supervivencia, la persona irá conformando el núcleo de su identidad -autoconcepto y autoestima- mediante el logro de las capacidades madurativas de orden psicosocial -esperanza, voluntad, propósito-. Estas servirán de coordenadas para situarse posteriormente a los requerimientos de la vida en los entornos externos al familiar. Así pues, se comprende el desarrollo y configuración de la propia identidad como un proceso eminentemente relacional. La identidad, pues, tiene que ver de modo notable con las relaciones interpersonales mantenidas desde las fases tempranas de la vida, lo cual presenta notables implicaciones para el ámbito familiar y escolar.

- Finalmente, y como conclusión general, la revisión llevada a cabo hace posible afirmar que la dimensión relacional humana permite hacerse a la persona completa. En efecto, en la apertura y donación al otro encuentra la persona el acabado de su identidad y su auténtica realización como plenitud de su personal vocación al amor. Así, se ha descrito que "toda persona nace como término de una relación personal, en el contexto de una relación personal [...] nace en el seno de una realidad amorosa" (Domínguez, 2007, p. 15) y toda su vida se verá orientada asimismo por las coordenadas interpretativas proporcionadas por este marco explicativo del origen. Somos, pues, por y para la relación interpersonal, para la comunión entre personas, esto es, para el amor. Relacionalidad e identidad personal constituye, pues, un binomio inseparable necesario 
para lograr una comprensión realista de la persona y su sentido existencial como vocación al amor.

\section{Referencias bibliográficas}

Altarejos, F. y Naval, C. (2004). Filosofía de la Educación. Navarra: EUNSA.

Aznar, J. y Tudela, J. (2017). "Aspectos biomédicos de la transexualidad", en J. Aznar (coord.): Transexualidad. Valoración pluridisciplinar del fenómeno y su regulación legal. Valencia: Universidad Católica de Valencia.

Bandura, A. (1982). Teoría del aprendizaje social. Madrid: Espasa Calpe.

Bowbly, J. (2006). Vínculos afectivos: formación, desarrollo y pérdida. Madrid: Morata.

Brizendine, L. (2007). El cerebro femenino. Barcelona: RBA.

Buber, M. (1949). ¿Qué es el hombre? México: Fondo de Cultura Económica.

Buber, M. (1969). Yo y tú. Buenos Aires: Nueva Visión.

De Gregorio (2007). Valores para vivir en una sociedad plural. Madrid: Fundación Universitaria Española.

Di Pietro, M.L. (2013). "El proceso de sexualización de la persona. El dato biológico", en L. Melina y S. Belardinelli (coords.): Amar en la diferencia. Las formas de la sexualidad y el pensamiento católico. B.A.C.: Madrid, pp. 265.

Domínguez, X.M. (2007). De todo corazón. Madrid: Fundación Enmanuel Mounier.

Erikson, E. (2000). El ciclo vital completado. Barcelona: Paidós Ibérica.

Fernández Mateos, M.L. (2005). El vínculo afectivo con el niño por nacer. Salamanca: Universidad Pontificia de Salamanca.

Isaacs, D. (1986). La educación de las virtudes humanas ( $8^{\mathrm{a}} \mathrm{ed}$.). Pamplona: Eunsa.

López Moratalla (2012). La identidad sexual: personas transexuales y con trastornos del desarrollo gonadal. Cuadernos de Bioética, XXIII(2), 347-371.

López Moratalla, N. (2007). Cerebro de varón y cerebro de mujer. Madrid: Rialp.

López Moratalla, N. (2008). La comunicación materno-filial durante el embarazo: el vínculo del apego. Pamplona: Eunsa.

L'Ecuyer, C. (2015). Educar en la realidad ( $2^{\mathrm{a} e d}$.). Barcelona: Plataforma Editorial.

Marías, J. (1995). Tratado de lo mejor. Madrid: Alianza editorial.

Millán Puelles, A. (1974). Economía y libertad. Madrid: Confederación de Cajas de Ahorro. 
Montagú, A. (2004). El tacto. La importancia de la piel en las relaciones humanas. Barcelona: Paidós.

Ortiz, E., Prats, J.I. y Arolas, G. (2014). La persona completa. Edicep: Valencia.

Pérez-Soba, J.J. (2005). "La pregunta por la persona. La respuesta de la interpersonalidad". Studia Theologica Matritensia, 3. Madrid: Facultad de Teología San Dámaso.

Polaino Lorente (2007). "El desarrollo de la identidad sexual en los varones: líneas de actuación en el tercer milenio". Revista Española de Pedagogía 238, 397-432.

Polaino Lorente (2008). "Identidad y diferencia: la construcción social de "género"", en J. de Irala (coord.): Mujer y varón. ¿Misterio o autoconstrucción? Madrid: Universidad Francisco de Vitoria, pp. 105-140.

Polaino Lorente, A. (2010). "La configuración de la identidad personal en la familia", en J. Cabanyes y M.A. Monge (eds.): La salud mental y sus cuidados. Pamplona: Eunsa, pp. 33-40.
Quaglia, R. y Vicente, F. (2007). "El papel del padre en el desarrollo del niño". INFAD Revista de Psicología 2, 167-182. http://infad.eu/RevistaINFAD/2007/n2/volu men1/0214-9877_2007_2_1_167-182.pdf

Real Academia Española (2019). https://dle.rae.es

Scola, A. (2002). Uomo-Donna. // "caso serio" del amore. Genova-Milano: Marietti.

Tizón, J.L. Tizón, J.L. (2016). “Crisis social y parentalidad líquida. Parte II". Temas de Psicoanálisis 11 7. http://www.temasdepsicoanalisis.org/wpcontent/uploads/2017/05/PARENTALIDADLIQUIDA-Y-CRISIS-SOCIAL.-PARTE-II.Jorge-L.pdf

Vasta, R., Haith, M.M. y Miller, S. (2001). Psicología infantil (2 ${ }^{\mathrm{a} e d .}$ ). Barcelona: Ariel.

Verny, T. (2011). El vínculo afectivo con el niño que va a nacer. Barcelona: Urano. 